\title{
Recherches sur Setaria labiatopapillosa (Perroncito, 1882) en Afrique occidentale
}

\section{III. - Infestation expérimentale de l'hôte normal et de différents hôtes anormaux}

\author{
par R. GIDEL * et J. BRENGUES **
}

O.C.C.G.E., Centre Muraz, Bobo-Dioulasso, Haute-Volta, O.R.S.T.O.M., 70 à 74, route d'Aulnay, F 93140 Bondy

\section{Résumé}

Infestation de l'hôte normal.

- La période prépatente a duré huit mois et une semaine.

- Au cours de cette période, une forte poussée d'éosinophilie est apparue un mois environ après l'infestation.

- Une légère éosinophilie s'est manifestée au moment présumé de la production des premières microfilaires.

- Au cours de la période d'état, des fluctuations de faible amplitude de la microfilarémie sanguine, liées à des variations de l'éosinophilie ont été observées.

- En fin d'expérimentation, l'absence passagère de réactions de l'hôte a permis une augmentation importante, mais transitoire, de la microfilarémie.

\section{Infestation des hôtes anormaux.}

- Ont été infestés: deux chèvres, un mouton, deux lapins, trois cobayes, cinq rats.

- Chez les rats et les cobayes une éosinophilie précoce mais légère est apparue; celle-ci a été plus tardive mais plus marquée chez les chèvres et le mouton; par contre elle n'a pas été observée chez les lapins.

- Deux rats et une chèvre sont décédés; un rat a présenté des troubles passagers. Ces décès et ces troubles semblent pouvoir être rapportés à l'infestation filarienne.

(*) Docteur Vétérinaire.

(**) Entomologiste médical de l'O.R.S.T.O.M. 


\section{Summary}

Infection of normal host. one week.

- The duration of prepatent period was eight months and

- During this time, an high increase in eosinophils occured about one month after infection.

- A slight eosinophilia was noticed at the presumed time of first microfilariae production.

- When microfilariae were found in the blood, slight fluctuations in microfilaremia, linked to changes in eosinophil count, were observed.

- At the end of experimentation, the momentary absence of vertebrate host reaction allowed an important but transient increase in microfilaremia.

\section{Infection of abnormal hosts.}

- Two goats, one sheep, two rabbits, three guinea-pigs and five rats have been infected.

- With rats and guinea-pigs, it appeared an early but slight eosinophilia; this one was later and more pronounced with goats and sheep; but it was not observed with rabbits.

- Two rats and one goat died. One rat was ill for a few days. It seems that these deaths and troubles may be associated with filarial infection.

\section{Introduction.}

Dans deux articles précédents (Brengues et al., 1969 ; Brengues et Gidel, 1972), nous avons indiqué les vecteurs de Setaria labiatopapillosa (Perroncito, 1882), étudié les variations de la microfilarémie et la dynamique des populations larvaires et imaginales de cette filaire, abordé l'infestation expérimentale de divers hôtes anormaux.

Au cours du présent travail, nous présenterons les résultats de l'infestation expérimentale de l'hôte normal (durée de la phase prépatente, variations de l'éosinophilie et de la microfilarémie sanguine) et de différents hôtes anormaux (variations de l'éosinophilie, troubles observés). Puis nous évoquerons les principales interactions parasitehôte vertébré susceptibles d'expliquer ces résultats et terminerons en situant notre étude dans le cadre général de l'épidémiologie des filarioses. 


\section{Infestation de l'hôte normal.}

\subsection{Matériel et méthodes.}

\subsubsection{Matériel biologiQue.}

Pour réaliser cette expérimentation, il était nécessaire de disposer d'une source de parasites, d'un vecteur et d'un hôte définitif (bovin) non infesté.

\section{La source de parasites.}

Nous avons dû rechercher un animal dont la microfilarémie sanguine était suffisamment élevée pour permettre l'infestation facile du vecteur. Comme nous l'avons $\mathrm{vu}$, cette condition est plus souvent remplie chez les jeunes animaux (Brengues et Gidel, 1972). A cet effet, une prospection a été entreprise dans plusieurs troupeaux de la région de Banfora (ville située à $85 \mathrm{~km}$ au sud-ouest de Bobo-Dioulasso). Seuls les animaux âgés de 18 mois ou moins étaient retenus et soumis à une prise de sang pour recherche et numération des microfilaires de $S$. labiatopapillosa. Cette numération était faite sur le terrain en utilisant des cellules de Nageotte, suivant la technique précédemment décrite (Brengues et al., 1969). Ainsi, nous avons pu sélectionner un taurin femelle, âgé de 15 mois environ, pesant $62 \mathrm{~kg}$, dont la microfilarémie sanguine était voisine de 4 microfilaires par $\mathrm{mm}^{3}$.

\section{Le vecteur.}

A la suite de Nelson (1962), nous avons constaté que Aedes aegypti L. est un bon vecteur expérimental pour S. labiatopapillosa (Brengues et al., loc. cit.). Dans ce travail, nous avons utilisé une souche originaire de Kongolikan (Haute-Volta), colonisée au laboratoire depuis de nombreuses années.

\section{L'hôte définitif.}

Il fallait avoir la certitude que cet animal ne soit pas déjà filarien. Dans ce but, nous avons choisi comme sujet un veau nouveau-né, dont la mère ne présentait pas de microfilaires sanguicoles, bien que ce critère soit insuffisant pour affirmer qu'elle n'hébergeait pas de filaires adultes (Brengues et Gidel, 1972). Cet animal était un taurin de sexe mâle.

\subsubsection{Méthodes de travaIL.}

Isolement de l'hôte définitif.

Il était capital de soustraire le veau au risque d'infestation accidentelle avant et pendant l'expérimentation. A cet effet, l'animal a été placé, dès la naissance, sous un abri moustiquaire de quatre mètres de côté, couvert d'un toit de tôle et ainsi conçu :

- sol et partie inférieure des côtés en ciment, surmontée d'une ossature métallique tendue de tulle moustiquaire;

- communication avec l'extérieur par un sas;

- alimentation en eau et évacuation des déchets à l'intérieur de l'abri. 
Ce local était entouré sur tout son périmètre par un fossé contenant de l'eau additionnée de pétrole et de DDT.

Le manœuvre chargé de l'entretien de l'animal (nourriture au lait artificiel en poudre) ne pénétrait que deux fois par jour dans le local. Au cours de chaque passage une pulvérisation d'insecticide à action immédiate (pyréthrines) était effectuée dans le sas.

\section{Choix du moment de l'infestation.}

L'hôte définitif, né le 11 janvier 1969, n'a été infecté que le 6 juin suivant. Ce délai, d'environ cinq mois, nous a paru nécessaire au cas où l'animal aurait été porteur d'anticorps filariens d'origine maternelle. Nous avons mis cette période à profit pour établir sa formule leucocytaire avant l'infestation (valeur moyenne sur huit numérations).

\section{Mode d'infestation.}

Le 6 juin au soir, 145 femelles d'A. aegypti porteuses de larves infestantes de S. labiatopapillosa ont été lâchées dans l'abri moustiquaire contenant le veau. La plupart des femelles ont été recapturées après deux nuits, soit le 8 juin au matin. Ensuite, une pulvérisation d'insecticide a été effectuée dans l'abri pour éliminer tout moustique ayant échappé à la capture.

Etude de la formule leucocytaire et recherche des microfilaires.

Pour ces examens, des prélèvements ont été effectués deux ou trois fois par semaine au cours du mois suivant l'infestation, puis une fois par semaine au cours des onze mois suivants, enfin tous les quinze jours jusqu'à la fin de l'expérimentation.

La formule leucocytaire a été établie de façon classique : pourcentage de chaque type de leucocytes établi sur 200 éléments examinés.

Pour la recherche des microfilaires, deux techniques ont été utilisées: recherche après concentration suivant la méthode de Sang et Petithory (1963); recherche sur quatre gouttes épaisses de $20 \mathrm{~mm}^{3}$, colorées au Giemsa, pendant trente minutes après déshémoglobinisation et fixation à l'alcool méthylique.

La méthode de concentration a été abandonnée dès l'apparition de microfilaires sur goutte épaisse de façon constante.

\subsection{RÉsultats.}

Chronologiquement, nous pouvons distinguer quatre étapes successives:

- infestation,

- période prépatente,

- date d'apparition des premières microfilaires sanguicoles,

- période d'état.

Ces différentes étapes sont traduites sur la figure 1 . 


\subsubsection{INFESTATION.}

Parmi les moustiques recapturés après les deux nuits passées dans l'abri moustiquaire, nous avons dénombré 32 femelles qui s'étaient gorgées sur le veau.

Par la dissection et l'examen des moustiques, nous avons pu déterminer:

- chez un lot témoin, le nombre moyen de filaires infestantes par moustique avant le repas, soit 3,8 filaires par femelle;

- le nombre total de filaires subsistant chez les femelles gorgées sur le veau, soit 25 filaires.

Ces données permettent d'estimer la quantité de filaires infestantes perdues par les 32 femelles au cours de leur repas de sang. Cette quantité est égale à : $(3,8 \times 32)$ $-25=97$ filaires.

Le nombre réel de filaires reçues par l'hôte peut être nettement inférieur à ce chiffre. En effet, celles-ci étant déposées sur la peau de l'hôte, certaines ne peuvent pénétrer. Ewert et Ho (1967) ont estimé cette perte à $45 \%$ du total des filaires déposées.

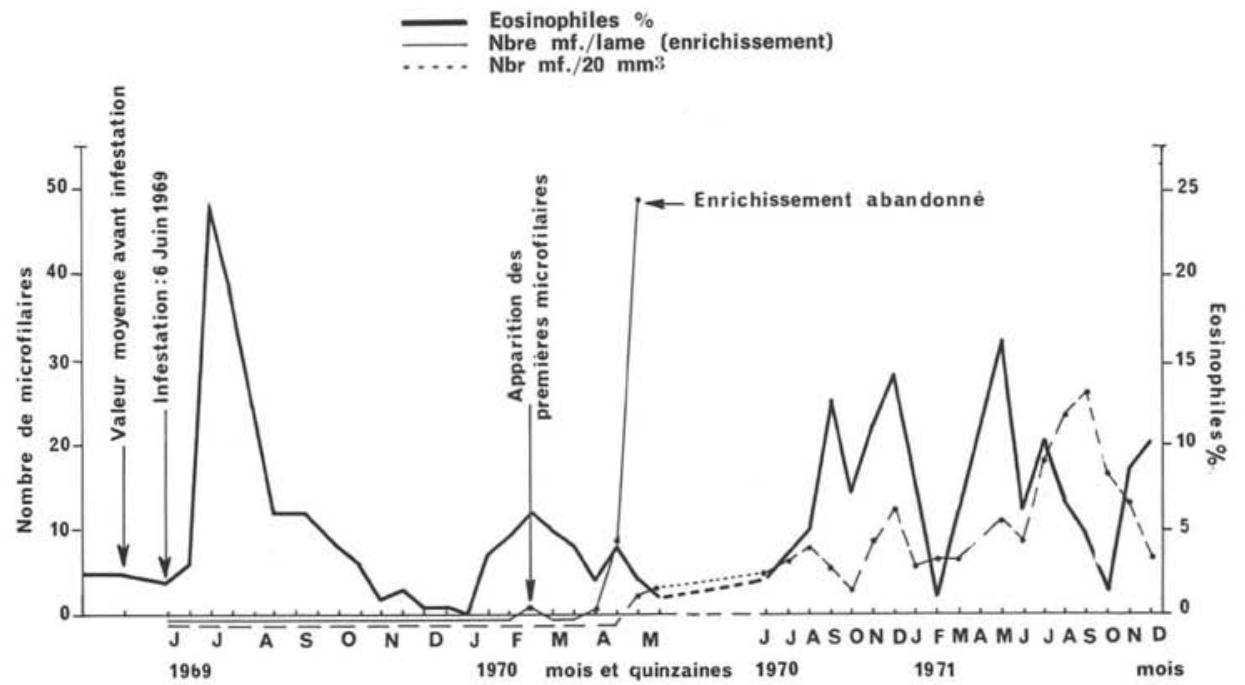

FIG. 1. - Variations de l'éosinophilie et de la microfilarémie chez l'hôte normal (bovin) infesté expérimentalement

\subsubsection{PÉRIOde PRÉPATENTE.}

Elle s'étend du jour de l'infestation (6 et 7 juin 1969) à la date d'apparition des premières microfilaires dans le sang (15 février 1970), soit huit mois et une semaine plus tard. Au cours de cette période, nous avons observé les variations suivantes de la formule leucocytaire : 
- Avant l'infestation, la formule moyenne s'établissait ainsi :

\begin{tabular}{|c|c|c|}
\hline Polynucléaires & neutrophiles $\quad \ldots \ldots \ldots \ldots \ldots \ldots \ldots \ldots$ & $34,3 \%$ \\
\hline$\gg$ & éosinophiles $\quad \ldots \ldots \ldots \ldots \ldots \ldots \ldots \ldots \ldots$ & $2,4 \%$ \\
\hline$\gg$ & basophiles $\ldots \ldots \ldots \ldots \ldots \ldots \ldots \ldots$ & $0,7 \%$ \\
\hline Mononucléaires & lymphocytes $\ldots \ldots \ldots \ldots \ldots \ldots \ldots \ldots$ & $57,2 \%$ \\
\hline » & $\ldots \ldots \ldots \ldots \ldots \ldots \ldots \ldots \ldots$ & 5,4 \\
\hline
\end{tabular}

- Du $13^{\circ}$ au $20^{\circ}$ jour qui suit l'infestation, nous avons noté un fort abaissement du rapport poly/mono, qui passe de 0,60 à 0,04 , dû à une diminution des polynucléaires neutrophiles et à une augmentation des lymphocytes.

- Entre le $20^{\circ}$ et le $25^{\circ}$ jour, la formule redevint normale.

- Le $27^{\circ}$ jour, on observa la montée brutale des éosinophiles passant de 4,5\% (au $25^{\circ}$ jour) à $25 \%$. Pendant le mois suivant, l'éosinophilie se maintint à un niveau élevé (fig. 1). Le rapport poly/mono a d'abord augmenté jusqu'à 1,2 sous l'action conjuguée de l'éosinophilie et d'une diminution des lymphocytes, puis jusqu'à 2,7 du fait d'une montée des polynucléaires neutrophiles au début de la décroissance de l'éosinophilie.

- A partir du troisième mois, la formule leucocytaire reprit progressivement son aspect normal au cours des deux mois suivants.

Dans la deuxième quinzaine de janvier, c'est-à-dire environ sept mois après l'infestation, on observa une légère recrudescence de l'éosinophilie (fig. 1).

\subsubsection{APPARItion DES PREMIÈres Microfilatres SANGUiCOLES.}

Les premières microfilaires sanguicoles ont été rencontrées, après concentration, le 15 février 1970, soit huit mois et une semaine après l'infestation. Jusqu'au début avril, les recherches furent à nouveau négatives. Puis le nombre de microfilaires dépistées après enrichissement augmenta régulièrement. A partir de mai, les gouttes épaisses devinrent positives ; l'enrichissement fut alors abandonné (fig. 1).

Il est remarquable de constater que l'apparition des premières microfilaires coïncide avec une petite poussée d'éosinophiles qui avait débuté un mois plus tôt (fig. 1).

\subsubsection{PÉRIOde D'ÉtAT.}

Nous avons suivi l'évolution de la formule leucocytaire et de la microfilarémie, non seulement chez le veau infesté expérimentalement, mais aussi chez l'animal infesté naturellement et utilisé comme source de parasites.

Chez l'animal infesté expérimentalement.

Après une phase d'éclipse en mars, nous avons noté la présence constante de microfilaires d'avril 1970 à décembre 1971 (fin de notre expérimentation). Durant 
les dix-sept premiers mois (jusqu'en septembre 1971), la microfilarémie augmenta. Cependant, cette progression ne se fit pas régulièrement, mais en dents de scie. Durant cette même période, l'éosinophilie subit des variations importantes dont les maximums demeurèrent cependant inférieurs au pic d'éosinophilie observé peu après l'infestation (fig. 1).

Il est intéressant de constater que les trois premiers pics de l'éosinophilie correspondent à de légers clochers de la microfilarémie. Il en est différemment de la dernière montée de la microfilarémie, la plus importante. Au cours de celle-ci, on observa une légère recrudescence de l'éosinophilie, puis une chute prononcée, alors que la microfilarémie continuait à augmenter. Ensuite, la microfilarémie décrut et, durant cette phase, s'amorça une nouvelle poussée d'éosinophilie (fig. 1).

A l'autopsie effectuée le 16 décembre 1971, on dénombra dans la cavité péritonéale six filaires adultes, dont quatre femelles et deux mâles.

\section{Chez l'hôte infesté naturellement.}

Chez celui-ci, au cours des vingt-huit mois d'observation, on nota une évolution dégressive mais irrégulière de la microfilarémie. Parallèlement, des variations sensibles de l'éosinophilie furent observées (fig. 2).

Comme pour l'animal précédent, on peut remarquer un certain synchronisme entre les pics de la microfilarémie et les légères poussées d'éosinophiles.

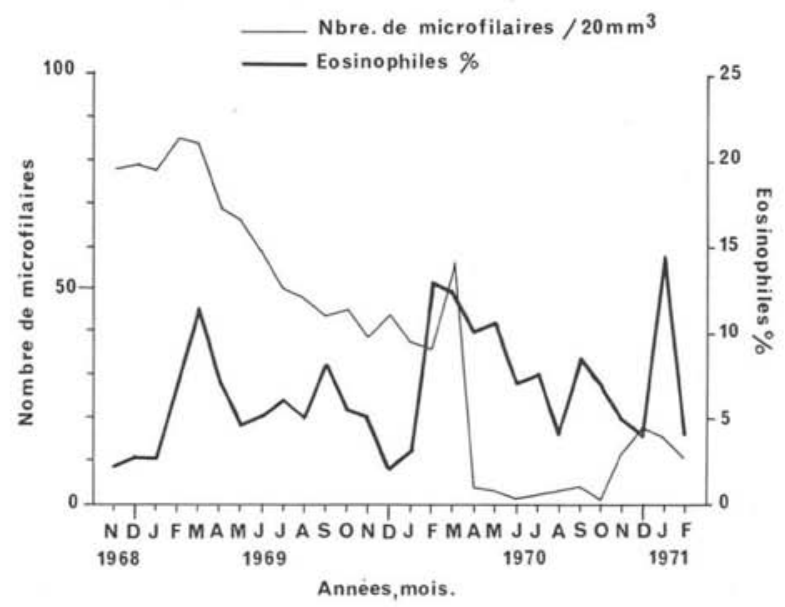

Fig. 2. - Variations de l'éosinophilie et de la microfilarémie, chez le bovin naturellement infesté, source de parasites

\subsection{Discussion.}

\subsubsection{Phase prépatente et apparition DES PREMiÈres Microfilaires.}

Les premières microfilaires sanguicoles ont été décelées environ huit mois après l'infestation. 
Au cours de cette période, on a enregistré deux poussées d'éosinophilie, l'une très importante apparaissant un mois après l'infestation, et l'autre, beaucoup plus faible, débutant un mois avant la détection des premières microfilaires.

La première poussée d'éosinophilie traduit vraisemblablement une réaction de l'hôte à un certain stade de la migration du parasite (phase tissulaire) ou de l'évolution de celui-ci (mue). Il est d'ailleurs possible que ces deux phénomènes agissent de façon concomitante. Cependant, il faut rappeler que cette hyperéosinophilie, très importante dans notre cas, n'est pas toujours observée dans les filarioses; ainsi, Edeson et al. $(1960 \mathrm{~b})$ ne relèvent pas d'augmentation notable des éosinophiles peu après l'infestation de quatre volontaires par Brugia malayi et B. pahangi.

La deuxième poussée, de faible amplitude, correspond à la production des premières microfilaires. De même, Edeson et al. (loc. cit.) observaient une éosinophilie marquée 10 à 15 semaines après l'infestation, soit au moment présumé de l'arrivée à maturité des filaires de Brugia. A la suite de Buckley (1958), ils estimaient que cette éosinophilie était une réponse à certaines substances produites par les vers matures. Le fait que les microfilaires aient été décelées dans le sang seulement un mois après le début supposé de la ponte peut s'expliquer, soit par leur extrême rareté, soit par leur absence dans la circulation sanguine.

Ce retard dans l'apparition des microfilaires dans le sang pourrait être motivé par l'existence d'une phase initiale de migration tissulaire, suivie ou non d'un blocage à un certain niveau. Ainsi, Duke (1960), transplantant des filaires adultes de Loa à des singes, constatait que les microfilaires n'apparaissaient dans le sang que deux ou trois semaines plus tard. Il estimait que ce délai pouvait être dû à un transit par le système lymphatique, suivi d'une accumulation des microfilaires dans les poumons. Si notre hypothèse est exacte, les filaires de $S$. labiatopapillosa atteindraient donc leur maturité sexuelle un mois avant la détection des premières microfilaires dans le sang, soit sept mois après l'infestation.

Enfin, nous pouvons comparer la durée de la phase prépatente à celle observée par différents auteurs pour d'autres filaires humaines et animales:

- Wuchereria bancrofti. Yokogawa (1939) décelait de rares microfilaires 82 et 111 jours après l'infestation expérimentale de deux volontaires. Cependant, pour les sujets naturellement infestés, on trouve exceptionnellement des microfilaires chez les enfants âgés de moins d'un an, bien que Jordan (1952) ait rencontré deux enfants positifs âgés de trois et six mois et que, plus récemment, Abdulcader et al. (1966) aient signalé la présence de microfilaires chez un sujet âgé de huit mois.

- Brugia malayi. Chez le chat, Edeson et Buckley (1959) ont rencontré des microfilaires dans le sang 75 à 100 jours après l'infestation. Chez le mérion, la phase prépatente dure de 79 à 116 jours (Ash et Riley, $1970 \mathrm{~b}$ ); chez le rat du coton et le hamster, elle dure de 3 à 4 mois (Edeson et al., 1962).

- Brugia pahangi. La période prépatente varie chez le chat et chez le mérion de 2 à 3 mois (Edeson et al., $1960 a$; Schacher, 1962 ; Ash et Riley, $1970 a$ ). 
- Loa. Chez les singes infestés (Duke, 1960), la période prépatente varie de 4 mois et demi (forme nocturne d'origine simienne) à 5 mois (forme diurne d'origine humaine).

- Onchocerca volvulus. Bien que Thomas et al. (1970) aient observé des microfilaires dermiques chez un sujet infesté 70 jours plus tôt, ce n'est qu'exceptionnellement que l'on trouve des microfilaires chez les sujets âgés de moins d'un an. Ainsi, le plus jeune sujet porteur de microfilaires observé par Philippon (com. pers.) était âgé de huit mois.

- Onchocerca flexuosa. Chez le cerf, des nodules contenant des filaires adultes peuvent apparaître moins de quatre mois après la naissance (Wenk, com. pers.).

- Dirofilaria immitis. Chez le chien, les microfilaires apparaissent dans le sang cinq à six mois après l'infestation (Kume et Itagaki, 1955).

- Litomosoides carinii. Chez le rat du coton, la durée de la période prépatente est de 50 jours au minimum (Scott et al., 1951) et de 65 jours en moyenne (Tanaka, 1964).

- Conispiculum flavescens. Menon et al. (1944) ont décelé les premières microfilaires entre 41 et 71 jours après l'infestation.

Il apparaît que pour certaines filaires (B. malayi et B. pahangi, L. carinii, C. flavescens et peut-être $W$. bancrofti et $O$. volvulus) la phase prépatente est courte, elle est inférieure ou sensiblement égale à trois mois. Pour d'autres filaires (Loa, D. immitis), cette phase est plus longue, elle est supérieure à quatre mois et demi. S. labiatopapillosa appartient donc à cette deuxième catégorie, la phase prépatente étant voisine de huit mois. Il est intéressant de noter que pour les sujets naturellement infestés, dépistés au cours de nos prospections, nous n'avons rencontré qu'exceptionnellement des microfilaires chez les animaux âgés de moins d'un an, le plus jeune sujet positif étant âgé de huit à dix mois.

\subsubsection{PÉRIODE D’ÉTAT.}

\section{Chez l'hôte expérimentalement infesté.}

Paradoxalement, elle débute par une phase d'éclipse au moment où la légère poussée d'éosinophilie, apparue avec les premières microfilaires, amorce sa décroissance. Les microfilaires réapparaissent dans le sang lorsque l'éosinophilie a recouvré son niveau normal. Ultérieurement, on observe des variations de la microfilarémie concomitante de poussées d'éosinophilie (fig. 1).

Il apparaît donc que les poussées d'éosinophilie expriment une réaction de l'hôte à la production ou à la migration des microfilaires. Cette réaction entraînerait soit une destruction, soit un blocage des microfilaires, s'opposant à leur libération dans la circulation générale, suivant différents mécanismes étudiés par plusieurs auteurs (in Brengues et Gidel, 1972). Le maintien de la microfilarémie à un niveau peu élevé et ses fluctuations de faible amplitude seraient donc une manifestation d'un processus de régulation et de défense de l'organisme. 
Cependant, ce processus peut présenter des défaillances transitoires comme en témoigne l'augmentation importante de la microfilarémie apparue en fin de période d'observation. Cette augmentation n'est pas concomitante d'une poussée notable d'éosinophilie. Celle-ci débute toutefois alors que la microfilarémie commence à décroître (fig. 1). Ainsi la réaction retardée de l'hôte aurait permis une augmentation importante, mais transitoire, de la microfilarémie sanguine.

Chez l'hôte naturellement infesté.

Nous avons noté une décroissance irrégulière de la microfilarémie sanguine. La courbe de celle-ci présente trois légers clochers synchrones de poussées d'éosinophilie (fig. 2). Cela confirme les résultats précédents et montre que la microfilarémie sanguine est sous la dépendance d'interactions hôte-parasite.

\section{Infestation d'hôtes anormaux.}

\subsection{MATÉRIEL ET MÉTHODEs.}

\subsubsection{Matériel Biologique.}

La source de parasites et le vecteur.

Nous avons utilisé la même source de parasites (veau filarien originaire de Banfora) et le même vecteur ( $A$. aegypti, souche de Kongolikan) que pour l'infestation expérimentale de l'hôte normal.

\section{Les animaux infestés.}

Nous avons infesté avec S. labiatopapillosa: deux chèvres, un mouton, deux lapins, trois cobayes et cinq rats. Les trois petits ruminants ont été séparés de leur mère peu après la naissance et isolés dans l'abri moustiquaire précédemment décrit (voir 2.1.2). Les dix rongeurs ont été placés dans la partie de l'animalerie réservée aux animaux sous expérimentation.

\subsubsection{Méthodes de travail.}

Choix du moment de l'infestation.

Les animaux ont été mis en observation au moins deux semaines avant l'infestation, et cette période a été mise à profit pour établir leur formule leucocytaire (valeur moyenne sur trois numérations ou plus).

\section{Mode d'infestation.}

Deux méthodes ont été utilisées soit séparément, soit, le plus souvent, conjointement : infestation par l'intermédiaire de l'insecte vecteur suivant la technique exposée précédemment (Brengues et al., 1969), inoculation sous-cutanée de filaires infestantes. 
Dans ce cas, les femelles infestantes d' $A$. aegypti étaient disséquées en eau physiologique. Les filaires étaient prélevées puis placées dans un verre de montre contenant quelques gouttes d'eau physiologique.·Enfin, elles étaient inoculées à l'aide d'une seringue sur la face dorsale, près de la colonne vertébrale, au niveau de la nuque ou des lombes.

Estimation du nombre de filaires reçues par l'hôte vertébré.

Les filaires inoculées par voie sous-cutanée étaient décomptées au moment de la dissection.

Pour estimer le nombre de filaires reçues par l'intermédiaire de l'insecte vecteur, nous avons eu recours à la méthode précédemment décrite (voir 2.2.1), soit : nombre escompté de filaires perdues par le vecteur $=$ (nombre moyen de filaires avant le repas - nombre moyen de filaires après le repas) $\times$ nombre de moustiques gorgés. Comme nous l'avons déjà dit, le nombre réel de filaires reçues par l'hôte vertébré peut être inférieur au nombre de filaires perdues par le vecteur, du fait de la perte possible de filaires sur la peau de l'hôte.

Etude de la formule leucocytaire et recherche des microfilaires.

La formule leucocytaire a été établie deux fois par semaine au cours du mois suivant l'infestation, puis une fois par semaine. Les microfilaires ont été recherchées uniquement chez les petits ruminants, zoologiquement voisins de l'hôte normal habituel. Nous avons utilisé la méthode de concentration précédemment citée (Sang et Petithory, 1963).

\subsection{Résultats.}

Les principaux résultats de ce travail sont portés au tableau I et à la figure 3 .

Chèvres.

Deux chèvres ont été infestées, l'une a reçu 152 et l'autre 268 filaires. Les deux animaux ont présenté une poussée d'éosinophilie qui a atteint son maximum trois semaines après l'infestation. Cette éosinophilie a ensuite diminué de façon irrégulière. Enfin, il faut noter une deuxième poussée apparue chez les deux animaux douze semaines après l'infestation (fig. 3).

Aucune microfilaire n'a été décelée durant la période d'observation (chèvre $\mathrm{n}^{\circ} 1$ : un an ; chèvre $\mathrm{n}^{\circ} 2$ : cinq mois).

Du point de vue clinique, la chèvre $\mathrm{n}^{\circ} 1$ n'a manifesté aucun trouble, par contre la chèvre $\mathrm{n}^{\circ} 2$ est décédée cinq mois après l'infestation en présentant une anémie marquée. Cependant, aucune lésion macroscopique n'a pu être décelée à l'autopsie.

\section{Mouton.}

Le mouton infesté a reçu 173 filaires. La formule leucocytaire est demeurée normale durant les cinq premières semaines, puis une forte éosinophilie est apparue à la sixième semaine. Cette éosinophilie a recouvré progressivement son niveau normal au 
Tableau I

Infestations des hôtes anormaux

\begin{tabular}{|c|c|c|c|c|c|c|}
\hline \multirow{2}{*}{$\begin{array}{l}\text { Animaux } \\
\text { infestés }\end{array}$} & \multirow{2}{*}{$\begin{array}{l}\text { Nombre } \\
\text { estimé de } \\
\text { filaires } \\
\text { inoculées } \\
\text { (1) }\end{array}$} & \multicolumn{2}{|c|}{ Infestation } & \multicolumn{2}{|c|}{ autopsie } & \multirow{2}{*}{ Observations } \\
\hline & & $\begin{array}{l}\text { mode } \\
(2)\end{array}$ & date & date & $\begin{array}{l}\text { résultats } \\
\text { (3) }\end{array}$ & \\
\hline chèvre $\mathrm{n}^{\circ} 1$ & 152 & $\begin{array}{l}\text { inf. nat. } \\
\text { et } \mathrm{s} / \mathrm{c}\end{array}$ & $28-1-68$ & - & 一 & $\begin{array}{c}\text { non } \\
\text { sacrifié }\end{array}$ \\
\hline chèvre $\mathrm{n}^{\circ} 2$ & 268 & inf. $s / c$ & 29-1-69 & $26-6-69$ & R.A.S. & décédée \\
\hline mouton & 173 & inf. $s / c$ & $4-2-69$ & - & - & non sacrifié \\
\hline lapin $\mathrm{n}^{\circ} 1$ & 39 & inf. $s / c$ & $26-12-68$ & 一 & 一 & 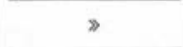 \\
\hline lapin $n^{\circ} 2$ & 83 & $\begin{array}{l}\text { inf. nat. } \\
\text { et } \mathrm{s} / \mathrm{c}\end{array}$ & $2-1-69$ & 一 & - & $》$ \\
\hline cobaye $n^{\circ} 1$ & 41 & $\begin{array}{l}\text { inf. nat. } \\
\text { et } s, c\end{array}$ & $29-12-68$ & $23-9-69$ & R.A.S. & sacrifié \\
\hline cobaye $n^{\circ} 2$ & 77 & $\begin{array}{l}\text { inf. nat. } \\
\text { et } \mathrm{s} / \mathrm{c}\end{array}$ & $6-1-69$ & $23-9-69$ & $\gg$ & 》 \\
\hline cobaye $n^{\circ} 3$ & 109 & $\begin{array}{l}\text { inf. nat. } \\
\text { et } \mathrm{s} / \mathrm{c}\end{array}$ & $8-1-69$ & $23-9-69$ & » & $\gg$ \\
\hline rat $\mathrm{n}^{\circ} \quad 1$. & 28 & $\begin{array}{l}\text { inf. nat. } \\
\text { et s/c }\end{array}$ & $30-12-68$ & $12-8-69$ & 》 & 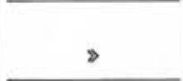 \\
\hline rat $\mathrm{n}^{\circ} 2$. & 28 & $\begin{array}{l}\text { inf. nat. } \\
\text { et } \mathrm{s} / \mathrm{c}\end{array}$ & $31-12-68$ & $12-8-69$ & 》 & 》 \\
\hline rat $\mathrm{n}^{\circ} 3$. & 49 & $\begin{array}{l}\text { inf. nat. } \\
\text { et } \mathrm{s} / \mathrm{c}\end{array}$ & $13-3-69$ & $19-8-69$ & 》 & $\gg$ \\
\hline rat $\mathrm{n}^{\circ} 4$ & 57 & $\begin{array}{l}\text { inf. nat. } \\
\text { et } \mathrm{s} / \mathrm{c}\end{array}$ & $6-1-69$ & $9-2-69$ & 》 & décédé \\
\hline rat $\mathrm{n}^{\circ} 5$ & 103 & $\begin{array}{l}\text { inf. nat. } \\
\text { et } s / c\end{array}$ & $7-1-69$ & $10-2-69$ & $\gg$ & 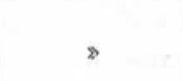 \\
\hline
\end{tabular}

(1) Le nombre réel de filaires reçues peut être inférieur au nombre estimé, du fait de la perte de filaires sur la peau de l'hôte au cours des infestations par vecteur.

(2) inf. nat. = infestation naturelle par vecteur ; inf.s/c. = inoculation par voie souscutanée.

(3) R.A.S. = Rien à signaler.

cours des cinq semaines suivantes. Ultérieurement, aucune autre poussée d'éosinophilie n'a été notée (fig. 3).

Aucune microfilaire n'a été décelée durant la période d'observation (un an). Au cours de cette même période, l'animal n'a présenté aucun trouble clinique. 


\section{Lapins.}

Deux lapins ont été infestés, l'un avec 83 et l'autre avec 39 filaires. Au cours de la période d'observation, aucun trouble clinique ni aucune modification significative de l'éosinophilie n'ont été notés (fig. 3).

Rats.

Cinq rats ont été infestés avec respectivement 28, 28, 49, 57 et 103 filaires.

Chez les trois premiers animaux, qui ont survécu, nous avons observé une légère poussée d'éosinophilie une semaine après l'infestation (fig. 3).

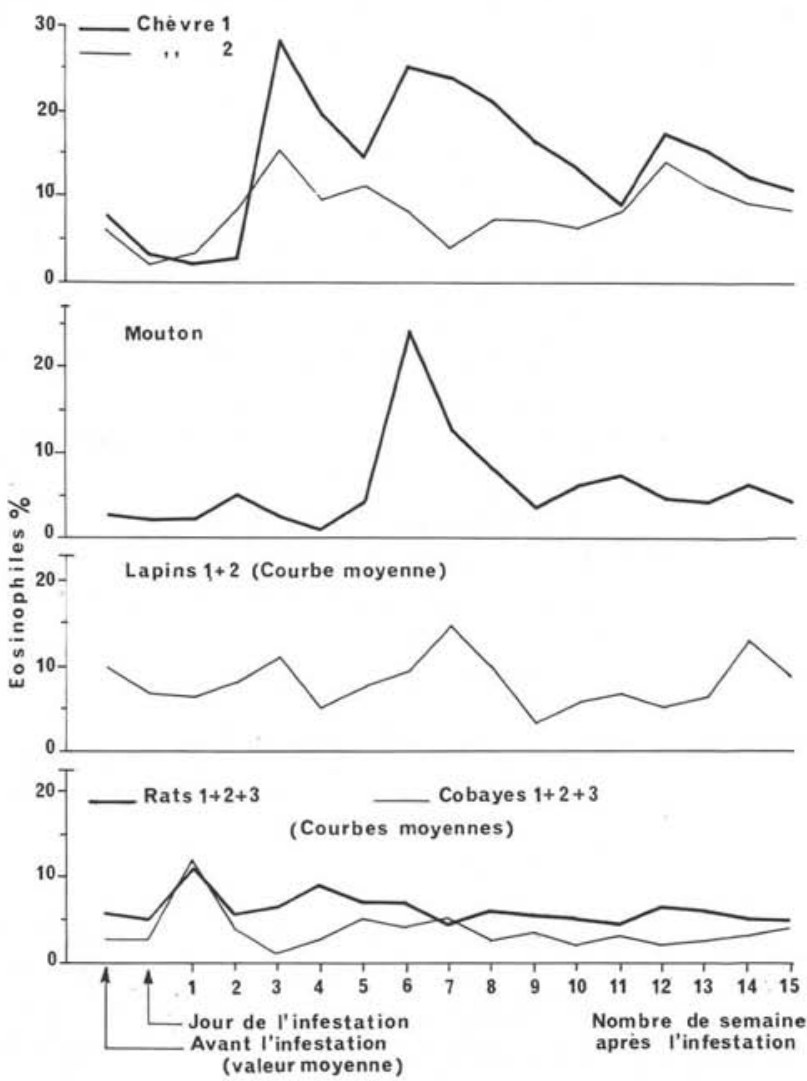

Fig. 3. - Variations de l'éosinophilie chez les hôtes anormaux infestés expérimentalement

Pour les deux animaux morts en cours d'expérimentation, l'un d'entre eux seulement (rat $\mathrm{n}^{\circ} 5$ ) a présenté une poussée d'éosinophilie débutant également une semaine après l'infestation. 
Trois des cinq rats ont présenté des troubles cliniques un mois après l'infestation. Le rat $\mathrm{n}^{\circ} 1$ a été malade pendant deux semaines (poils hérissés, aspect abattu, cachexie), puis s'est rétabli. Les rats $\mathrm{n}^{\text {os }} 4$ et 5 sont décédés brutalement, sans aggravation apparente de leur état. L'autopsie de ces animaux n'a pas permis de déceler de lésion macroscopique. Il est à remarquer que ces animaux avaient reçu la plus grande quantité de filaires.

\section{Cobayes.}

Trois cobayes ont été infestés avec respectivement 41, 77 et 109 filaires. Comme les rats, ils ont présenté une éosinophilie précoce une semaine après l'infestation (fig. 3). Aucun signe clinique n'a été observé au cours de la période d'observation, et les autopsies effectuées huit et demi et neuf mois plus tard n'ont révélé aucune lésion apparente.

\subsection{Discussion.}

\subsubsection{MOdificAtion DE LA FORMUle LeUCOCYTAIRE.}

Exception faite des lapins et d'un rat, tous les autres animaux infestés ont présenté une ou plusieurs poussées d'éosinophilie au cours des semaines qui ont suivi l'infestation. L'augmentation de l'éosinophilie a été précoce chez les rats et les cobayes (une semaine après l'infestation), plus tardive chez les chèvres et le mouton (respectivement trois et six semaines après l'infestation). Il est à remarquer l'apparition d'une deuxième poussée d'éosinophilie chez les deux chèvres douze semaines après l'infestation.

Bien qu'aucune microfilaire n'ait pu être décelée dans le sang, l'observation des courbes de l'éosinophilie chez les trois petits ruminants donne à penser que les filaires infestantes ont vraisemblablement accompli une certaine évolution. Cela expliquerait la rencontre exceptionnelle de filaires péritonéales chez les petits ruminants, comme ont pu l'observer, au Tchad, Graber (1968), et en Haute-Volta, à Bobo-Dioulasso, Ouedraogo (com. pers.), et à Banfora, Kirakoye (com. pers.).

A ce propos, il convient aussi de rappeler les travaux de Danaraj et al. (1957) et Buckley (1958) qui montraient que certaines filaires animales inoculées à un hôte anormal pouvaient provoquer un syndrome d'éosinophilie pulmonaire tropicale.

\subsubsection{Pathogénicité.}

Nous n'avons observé chez aucun animal de signes nerveux comparables à ceux décrits au Japon par Itagaki (1958). Cependant, parmi les treize animaux inoculés, trois sont décédés et un quatrième a présenté des troubles sérieux mais passagers.

Bien que l'autopsie n'ait pas permis de confirmer l'étiologie filarienne de ces décès, il est raisonnable de la suspecter, étant donné que chez les animaux des mêmes espèces, maintenus dans les mêmes conditions à l'animalerie à cette époque, aucune mortalité n'a été enregistrée.

Il est à remarquer que la chèvre la plus infestée est décédée mais qu'elle a survécu cinq mois à l'infestation. Cette survie relativement longue pourrait s'expliquer par le fait que, chez cet animal, le parasite peut accomplir une certaine évolution (voir 3.2.1). 
Par contre, chez les deux rats décédés, la survie a été plus courte et identique (un mois dans les deux cas). Les troubles passagers observés chez le rat $\mathrm{n}^{\circ} 1$ sont également apparus un mois après l'infestation. Enfin, il est à noter que les deux animaux décédés avaient reçu la plus forte infestation.

Le fait que les trois animaux qui sont morts en cours d'expérimentation étaient les sujets les plus infestés de leur espèce renforce la probabilité d'une étiologie filarienne avancée précédemment.

\section{Conclusion.}

Ce travail a d'abord permis d'évaluer exactement la durée de la période prépatente de la sétariose, c'est-à-dire la période qui s'écoule entre l'infestation et l'apparition dans le sang des premières microfilaires. Dans le cas de notre expérimentation, cette durée est égale à huit mois et une semaine. D'autre part, nous avons pu constater que la durée de l'évolution chez le vecteur $(A$. aegypti) variait de huit à quatorze jours en fonction de la température. Cette observation est voisine de celle de Nelson (1962). Connaissant ces deux données, on en déduit que la durée totale et minimum du cycle est donc égale à huit mois et demi; la majeure partie de celui-ci $(97 \%$ de la durée totale) s'effectuant chez l'hôte vertébré.

Nous avons aussi constaté que l'évolution de la microfilarémie sanguine n'était pas un phénomène simple dépendant uniquement de la production et de la mortalité des microfilaires. En effet, nous avons observé des fluctuations de la microfilarémie sanguine liées à des réactions de l'hôte exprimées par des variations de l'éosinophilie.

Les résultats du présent travail et de l'étude précédente (Brengues et Gidel, 1972) nous permettent de constater que les sujets infestés peuvent réagir de façon très variable au parasite en fonction de différents facteurs.

On peut distinguer trois groupes de sujets :

- Animaux totalement réfractaires au parasite. Le fait que nous ayons trouvé, quel que soit l'âge, une fraction importante de la population ne présentant ni filaires adultes, ni microfilaires (Brengues et Gidel, loc. cit.) permet de penser que certains sujets sont totalement réfractaires au parasite, présentant une immunité naturelle ou acquise.

- Animaux partiellement réfractaires au parasite. Nous avons constaté que certains animaux porteurs d'un grand nombre de filaires adultes ne présentaient pas de microfilaires sanguicoles (Brengues et Gidel, loc. cit.). Il est possible que certains de ces sujets supportent l'évolution du parasite jusqu'au stade adulte, mais ne permettent pas l'établissement d'une microfilarémie sanguine.

- Animaux sensibles au parasite. Chez ceux-ci, les filaires adultes produisent des microfilaires que l'on retrouve dans le sang. Cependant, la microfilarémie de ces animaux subit des variations liées aux réactions de l'hôte. Celles-ci peuvent être provoquées soit par les parasites déjà en place, avec pour conséquence une régulation de 
la microfilarémie sanguine (voir 2.3.2), soit par les réinfestations qui entraîneraient un abaissement transitoire de la microfilarémie (Brengues et Gidel, loc. cit.).

En conséquence, s'il semble permis d'apprécier l'incidence de la filariose, au niveau d'une population, en se basant sur la fréquence et l'abondance des microfilaires sanguicoles, il est, par contre, hasardeux d'estimer l'existence et le degré du parasitisme chez un individu à partir de la seule parasitémie sanguine.

Enfin, chez la plupart des hôtes anormaux, nous avons observé une éosinophilie apparaissant dans les semaines qui suivent l'infestation. De plus, trois animaux, les plus infestés de leur groupe, ont succombé. Bien que nous n'ayons pas de preuve irréfutable, ces décès semblent pouvoir être rapportés à l'infestation filarienne. Ceci montre le rôle pathogène susceptible d'être exercé par une filaire en impasse parasitaire chez un hôte anormal. Rappelons à ce propos que plusieurs auteurs ont démontré le pouvoir pathogène de différentes filaires inoculées à des hôtes anormaux (Itagaki, 1958 ; Shoho, 1959) et que l'homme peut être l'un deux (Danaraj et al., 1957 ; Buckley, 1958 ; différents auteurs in Euzeby, 1964).

Etant donné que $S$. labiatopapillosa est transmise par divers culicidés dont certaines espèces au moins (notamment, Mansonia uniformis) sont très agressives vis-à-vis de l'homme, des larves infestantes sont donc inoculées à celui-ci. A la suite de Nelson (1962), et compte tenu de nos observations, on peut donc penser que cette filaire est susceptible de provoquer chez l'homme divers troubles associés à une éosinophilie, en particulier chez les éleveurs vivant en contact étroit avec leur cheptel.

\section{REMERCIEMENTS}

Nous tenons particulièrement à remercier, pour l'aide ou les conseils qu'ils nous ont apportés : Dioulasso.

- M. Séré, médecin-vétérinaire, chef du service de la production animale à Bobo-

- M. R. Le Berre, directeur de recherches de l'O.R.S.T.O.M., chef de la section onchocercose du Centre Muraz.

- M. P. Sales, adjoint au chef du service de documentation de l'O.C.C.G.E.

- M. B. Athawet, infirmier-spécialiste, laboratoire de biologie du Centre Muraz.

\section{Bibliographie}

Abdulcader (M. H. M.), Rajakone (P.), Rajendran (K.) et Aponso (L.), 1966. - Age, sex and house distribution of Wuchereria bancrofti microfilaraemia in Ceylon. Amer. J. trop. Med. Hyg., 15 (4), 519-522.

AsH (L. R.) et Riley (J. M.), $1970 a$. - Development of Brugia pahangi in the jird, Meriones unguiculatus, with notes on infections in other rodents. J. Parasit., 56 (5), 962 968. 
- et - $1970 \mathrm{~b}$. - Development of subperiodic Brugia malayi in the jird, Meriones unguiculatus, with notes on infections in other rodents. J. Parasit., 56 (5), 969973.

Brengues (J.), Gidel (R.) et Rodhain (F.), 1969. - Recherches sur Setaria labiato-papillosa (Perroncito, 1882) en Afrique occidentale. I. Vecteurs naturels et vecteur expérimental, étude des variations de la microfilarémie, infestations d'hôtes anormaux. Ann. Parasit. hum. comp., 44 (5), 625-640.

- et -, 1972. - Recherches sur Setaria labiatopapillosa (Perroncito, 1882) en Afrique occidentale. II. Dynamique de cette filariose dans les conditions naturelles. Ann. Parasit. hum. comp., 47, (4), 597-611.

BUCKLEY (J. J. C.), 1958. - Occult filarial infections of animal origin as a cause of tropical pulmonary eosinophilia. E. Afr. Med., J., 35 (8), 493-500.

Danaraj (T. J.), Da Silva (L.S.) et Schacher (J.F.), 1957. - The filarial complementfixation test in eosinophilic lung (tropical eosinophilia). A preliminary report. Proc. Alumni Ass. Malaya, 10 (2), 109-116.

DUKE (B. O. L.), 1960. - Studies on loiasis in monkeys. II. The population dynamics of the microfilariae of Loa in experimentally infected drills (Mandrillus leucophaeus). Ann. trop. Med. Parasit., 54 (1), 15-31.

Edeson (J. F. B.) et BuckLey (J. J. C.), 1959. - Studies on filariasis in Malaya on the migration and rate of growth of Wuchereria malayi in experimentally infected cats. Ann. trop. Med. Parasit., 53 (1), 113-119.

-, Ramachandran (C. P.), Zaini (M. A.), Nair (S.) et Kershaw (W.F.), 1962. - The transmission of Malayan filariasis to rodents. Trans. R. Soc. trop. Med. Hyg., 56 (4), 269.

-, Wharton (R. H.) et Laing (A. B. G.), 1960 a. - A preliminary account to the transmission, maintenance and laboratory vectors of Brugia pahangi. Trans. R. Soc. trop. Med. Hyg., 54 (5), 439-449.

-, Wilson (T.), Wharton (R. H.) et Laing (A. B. G.), $1960 \mathrm{~b}$. - Experimentally transmission of Brugia malayi and B. pahangi to man. Trans. R. Soc. trop. Med. Hyg., 54 (3), 229-234.

EuzÉBy (J.), 1964. - Les zoonoses helminthiques. Vigot frères. Editeurs, Paris, 389 pp.

EWERT (A.) et Ho (B. C.), 1967. - The fate of Brugia pahangi larvae immediately after feeding by infective vector mosquitoes. Trans. R. Soc. trop. Med. Hyg., 61, (5), 659-662.

Graber (M.), 1968. - in : Bilan d'activités. T. III, helminthes et helminthiases. I.E.M.V.T. Alfort, France, 192 pp.

ITAGAKI (S.), 1958. - La filariose chez les animaux domestiques au Japon. I. La paralysie lombaire chez la chèvre, le mouton et le cheval. Off. Intern. Epizoot., 49 bis, 526-530.

Jordan (P.), 1952. - Wuchereria bancrofti. Correspondence to the Editor. Trans. R. Soc. trop. Med. Hyg., 46 (2), 207-208.

Kume (S.) et ItAgaki (S.), 1955. - On the life-cycle of Dirofilaria immitis in the dog as the final host. Br. Vet. J., 111 (1), 16-24. 
Menon (T. B.), Ramamurti (B.) et Rao (D. S.), 1944. - Lizard filariasis. An experimental study. Trans. R. Soc. trop. Med. Hyg., 37 (6), 373-386.

Nelson (G.S.), 1962. - Observations on the development of Setaria labiato-papillosa using new techniques for infecting Aedes aegypti with this nematode. J. Helminth., 38 (3), 281-296.

SANG (H. T.) et Petithory (J.), 1963. - Techniques de concentration des microfilaires sanguicoles. Bull. Soc. Path. exot., 56 (3), 197-206.

SChaCHer (J.F.), 1962. - Developmental stages of Brugia pahangi in the final host. J. Parasit., 48 (5), 693-706.

Scott (J. A.), Macdonald (E. M.) et Terman (B.), 1951. - A description of the stages in the life cycle of the filarial worm Litomosoides carinii. J. Parasit., 37 (5), sect. I, 425-432.

Sноно (C.), 1959. - Sur les filaires chez les Equidés et les Bovidés. Rev. Elev. Méd. vét. Pays tropicaux, 12 (1), 43-52.

TANAKA (H.), 1964. - « Observations on the cotton rat as a laboratory animal for experimental filariasis studies. II. The course of infections with Litomosoides carinii ». Jap. J. Parasit., 13 (6), 507-513.

Thomas (T.), Chastel (C.) et Forcain (L.), 1970. - Latence clinique et parasitaire dans les filarioses à Loa loa et à Onchocerca volvulus. Bull. Soc. Path. exot., 63 (1), 90-94.

Yokogawa (S.), 1939. - Transmission of Wuchereria bancrofti. Trans. R. Soc. trop. Med. Hyg., 33, 363-364. 